\title{
常导低速磁悬浮列车弯道承载能力计算
}

\author{
崔鹏*，李杰，刘德生 \\ 国防科技大学三院磁悬浮工程技术研究中心, 长沙 410073 \\ *E-mail: cpthree@sina.com
}

收稿日期: 2009-03-08; 接受日期: 2009-08-28

“十一五”国家科技支撑计划重点课题(批准号: 2006BAG02B05-04)和国家自然科学基金(批准号: 60404003)资助项目

\begin{tabular}{l|l|l} 
摘要 针对磁悬浮列车位于弯道上时电磁铁与轨道的错位情况, 以磁悬浮列车单转向架 & 关键词 \\
为研究对象, 利用磁通管法得到悬浮模块提供的悬浮力和导向力计算公式. 根据列车轨道 & 低速磁悬浮列车 \\
超高条件下的动力学平衡条件, 分析了影响磁悬浮列车弯道承载能力的几个主要因素, 最 & 弯道 \\
终给出列车承载能力的定量计算公式. & 磁通管 \\
承载能力
\end{tabular}

常导电磁悬浮(EMS)型低速磁悬浮交通系统具 有噪音小、无污染、安全舒适、爬坡能力强、转弯半 径小等优点, 受到了世人越来越多的关注和青睐, 被 誉为二十一世纪城市内部和城市与市郊卫星城之间 的新型交通系统 ${ }^{[1]}$. EMS 型磁悬浮列车依靠电磁力支 撑车辆, 由于电磁铁的铁芯材料存在磁饱和特性, 电 磁铁可以提供的电磁力有限. 当磁悬浮列车位于弯 道段时, 电磁铁与轨道之间发生水平错位, 导致最大 电磁力减小. 因此, 列车在弯道段的承载能力比平直 段小, 这是提高磁悬浮列车承载能力的瓶颈. 日本爱 知世博会上投入商业运营的 Linimo 就曾经因为悬浮 系统在水平弯道段的承载能力不足而导致列车无法 正常悬浮和运行 ${ }^{[1]}$. 要推动 $\mathrm{EMS}$ 型低速磁悬浮列车 的工程化进程, 确保列车运行的可靠性和安全性, 对 其承载能力特别是在弯道段的承载能力进行定量研 究是非常必要的. 国内外相关研究处于起步阶段, 未 见相关成果的报道. 本文首先推出悬浮模块在弯道 段的电磁力计算公式, 并根据列车轨道超高条件下 的动力学平衡条件, 分析了影响列车弯道承载能力 的几个因素, 从而得到了列车弯道承载能力的计算
公式.

\section{1 悬浮系统弯道段承载能力问题描述及简化}

整列磁悬浮列车的承载能力可以通过计算单转 向架的承载能力类推得到 ${ }^{[1,2]}$. 单转向架是 EMS 型低 速磁悬浮列车的基本结构单元和功能单元, 其结构 如图 1 所示. 转向架由两个悬浮模块组成. 悬浮模块 由箱梁、悬浮电磁铁和间隙传感器等部分组成. 两个

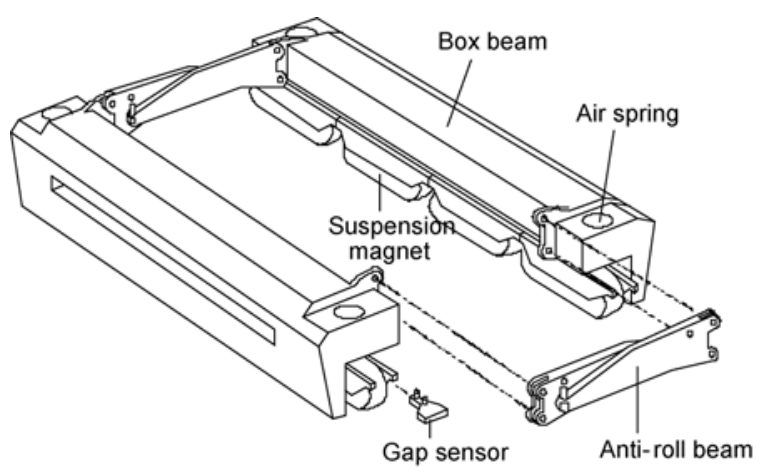

图 1 转向架结构示意图 
悬浮模块之间是通过防侧滚梁连接在一起的. 车体 的质量通过空气弹簧作用在悬浮模块两端.

下面以单转向架系统为对象, 从悬浮系统在弯 道段的电磁力和轨道超高条件下列车的动力学平衡 条件两方面入手, 研究悬浮系统在水平弯道段的承 载能力问题. 由于横向止档的存在, 在轨道的弯道段 模块与轨道之间的相对位置被限制在如图 2 所示的 范围内. 设弯道半径为 $R$, 轨道两个极面之间的距离 为 $p$, 模块长度的一半为 $L$, 轨道的轨距为 $2 D$. 在列 车通过弯道段时, 两个模块的转弯半径相差 $2 D$, 由 于 $R \gg 2 D$, 因此在后续有关承载能力的讨论中, 认 为两个模块的磁极相对轨道的错位情况完全一致. 采用 $T_{1}, T_{2}, L_{1}, L_{2}$ 描述模块的一个磁极与半径为 $R$ 的 轨道极面的相对位置关系, 如图 2 所示.

\section{2 悬浮系统在弯道段的电磁力计算}

设悬浮间隙为 $\delta$, 电磁铁与轨道极面的错位为 $x$, 电磁铁线圈的匝数为 $N$, 电感为 $L_{\mathrm{m}}$, 悬浮电流为 $i$, 气 隙的磁场能量为 $E_{\mathrm{m}}$, 气隙的磁导为 $G$, 气隙磁阻为 $R_{\mathrm{m}}$, 磁路的磁通为 $\Phi$, 磁感应强度为 $B$, 平均截面积 为 $\bar{S}$, 磁阻平均长度为 $L_{p}$, 磁势为 $\Psi$, 根据电磁学原 理 ${ }^{[1]}$, 有

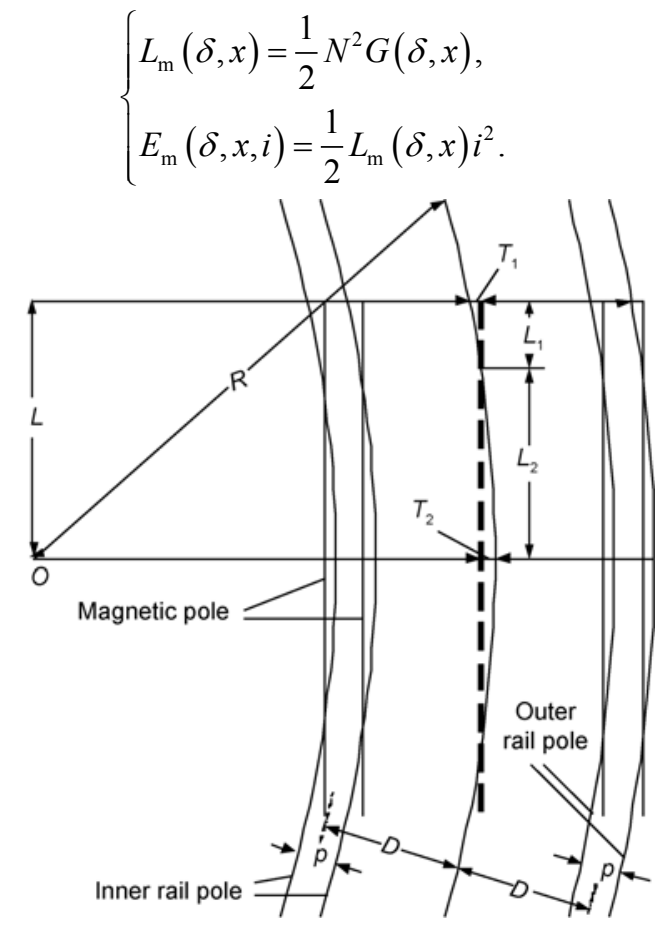

图 2 转向架模块与轨道的错位示意图
且 $\Phi=B \bar{S}, \Psi=N i=\Phi R_{\mathrm{m}}, R_{\mathrm{m}}=1 / G=L_{p} /\left(\mu_{0} \bar{S}\right)$, 真 空磁导率为 $\mu_{0}$. 悬浮电磁力和导向电磁力可表示为

$$
\left\{\begin{aligned}
F_{\mathrm{L}} & =-\frac{\partial E_{\mathrm{m}}(\delta, x, i)}{\partial \delta}=-\frac{N^{2} i^{2}}{2}\left[\frac{\partial}{\partial \delta} G(\delta, x)\right] \\
& =-\frac{2 \delta^{2} B^{2}}{\mu_{0}^{2}}\left[\frac{\partial}{\partial \delta} G(\delta, x)\right], \\
F_{\mathrm{G}} & =-\frac{\partial E_{\mathrm{m}}(\delta, x, i)}{\partial x}=-\frac{N^{2} i^{2}}{2}\left[\frac{\partial}{\partial x} G(\delta, x)\right] \\
& =-\frac{2 \delta^{2} B^{2}}{\mu_{0}^{2}}\left[\frac{\partial}{\partial x} G(\delta, x)\right] .
\end{aligned}\right.
$$

因此, 只需计算出气隙磁导 $G(\delta, x)$, 就可以根据 (2)式推导出悬浮电磁力和导向电磁力的表达式.

磁通管法是计算空间磁导的一种常用方法 ${ }^{[3 \sim 6]}$. 若磁通管的平均长度为 $L_{p}$, 平均截面积为 $S_{p}$, 则磁通 管的磁导为 $G=\mu_{0} S_{p} / L_{p}$. 考虑到磁通管中的磁通分布 不均匀, 将磁通管分成无数个体积元 $\mathrm{d} V$, 积分得到 磁通管磁导

$$
G=\mu_{0} \iiint_{V} \frac{\mathrm{d} V}{L_{p}^{2}} .
$$

下面采用磁通管法计算模块悬浮系统的磁路磁 通和电磁力. 将模块分为 I, II, III, IV 四个区间, 各个 区间产生的悬浮力的大小为 $F_{1_{\perp} \mathrm{I}}, F_{1_{\perp I I}}, F_{1_{\perp} \mathrm{III}}$ 和 $F_{1_{\perp I \mathrm{IV}}}$, 导 向力的大小为 $F_{\mathrm{g}_{\mathrm{I}} \mathrm{I}}, F_{\mathrm{g}_{\mathrm{II}} \mathrm{I}}, F_{\mathrm{g} \_\mathrm{III}}$ 和 $F_{\mathrm{g} \_\mathrm{IV}}$, 如图 3 所示.

通常情况下, 区间 I, II 所提供的悬浮力和导向力 分别和区间 IV, III 所提供的悬浮力和导向力一致, 即

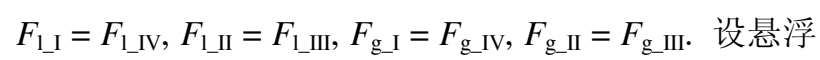
力合力 $F_{1}$ 的正方向为垂直于电磁铁极面向上, 导向 力合力 $F_{\mathrm{g}}$ 的正方向为平行于电磁铁极面指向弯道外 侧, 则

$$
\left\{\begin{aligned}
F_{1} & =F_{1 \_\mathrm{I}}+F_{1 \_\mathrm{II}}+F_{1 \_\mathrm{II}}+F_{1 \_\mathrm{IV}} \\
& =2\left(F_{1 \_\mathrm{I}}+F_{1 \_\mathrm{II}}\right), \\
F_{\mathrm{g}} & =-F_{\mathrm{g} \_\mathrm{I}}+F_{\mathrm{g} \_\mathrm{II}}+F_{\mathrm{g} \_\mathrm{III}}-F_{\mathrm{g} \_\mathrm{IV}} \\
& =2\left(F_{\mathrm{g} \_\mathrm{II}}-F_{\mathrm{g} \_\mathrm{I}}\right) .
\end{aligned}\right.
$$

考虑到实际的轨道弯道半径 $R$ 相对 $L_{1}$ 和 $L_{2}$ 足够 大, 为了便于计算, 将每个区间内的轨道曲线近似为 直线. 首先分析区间 I. 按照前述的磁通管划分原则, 将区间 I 电磁铁靠弯道内侧的磁极与轨道之间的磁通 划分为三个磁通管, 如图 4(a)所示, 其中 $h$ 为悬浮间隙. 


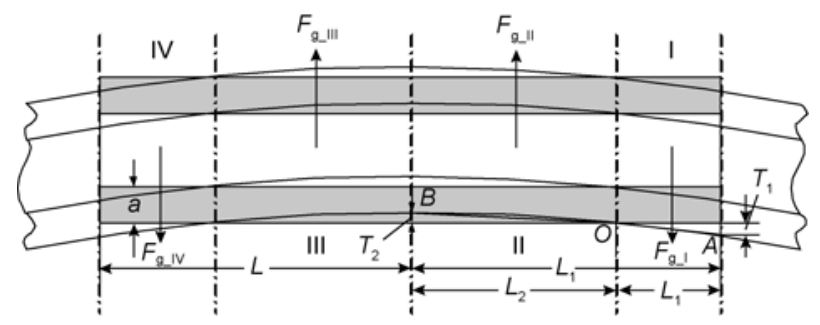

图 3 模块与轨道的相对位置

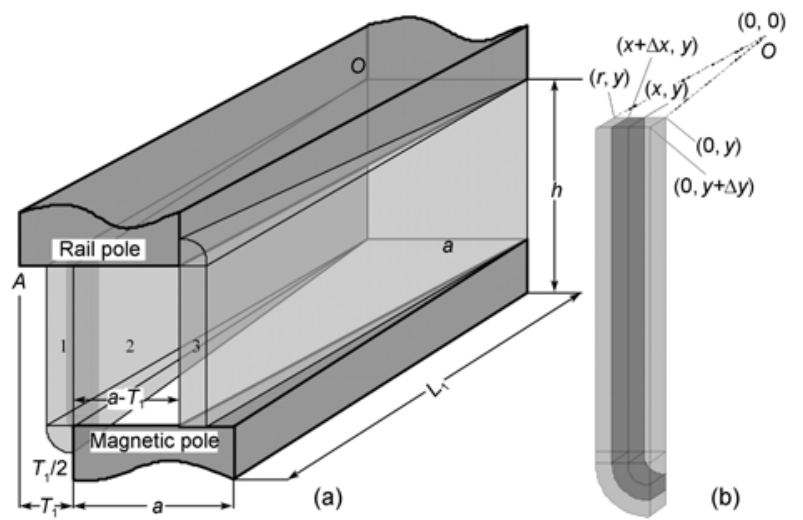

图 4 单侧磁极区间 I 的磁通管划分

(a) 磁通管的划分; (b) 体积微元

对于磁通管 1, 体积微元如图 4(b)所示, 有

$$
\left\{\begin{array}{l}
\mathrm{d} V_{1}=\left(\frac{\pi}{2} x+h\right) \mathrm{d} x \mathrm{~d} y, \\
L_{p}=\frac{\pi}{2} x+h, \\
r=\frac{T_{1}}{2 L_{1}} y .
\end{array}\right.
$$

根据(3)式，磁通管 1 的磁导为

$$
\begin{aligned}
G_{1} & =\mu_{0} \int_{0}^{L_{1}} \int_{0}^{r} \frac{\mathrm{d} V_{1}}{L_{p}^{2}} \\
& =\frac{2 \mu_{0}}{\pi} \int_{0}^{L_{1}} \ln \left(1+\frac{\pi r}{2 h}\right) \mathrm{d} y \\
& =\frac{2 \mu_{0} L_{1}}{\pi^{2} T_{1}}\left(\left(4 h+\pi T_{1}\right) \ln \left(1+\frac{\pi T_{1}}{4 h}\right)-\pi T_{1}\right) .
\end{aligned}
$$

极面宽度 $a \ll R$, 因此磁通管 3 与磁通管 1 的形 状以及磁通分布基本一致, 即 $G_{3} \approx G_{1}$. 对于磁通管 2 , 根据 $G=\mu_{0} S_{p} / L_{p}$ 有

$$
G_{2}=\mu_{0} \frac{S_{p}}{h}=\frac{\mu_{0} L_{1}}{2 h}\left(2 a-T_{1}\right) .
$$

结合(6)和(7)式，得到区间 I 电磁铁靠的极面与 轨道之间的磁导 $G_{\mathrm{I} \mathrm{a}}=G_{1}+G_{2}+G_{3}$. 靠弯道外侧的 磁极与轨道之间的磁导与弯道内侧的磁导相同, 即

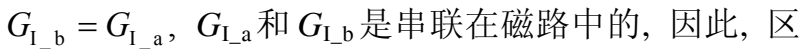
间 I 磁路的磁导为 $G_{\mathrm{I}}=G_{\mathrm{I} \mathrm{a}} / 2$.

根据(2)式，计算得到区间 I 电磁铁产生的悬浮力 $F_{L_{-} \mathrm{I}}$ 和导向力 $F_{\mathrm{g}_{-} \mathrm{I}}$

$$
\left\{\begin{aligned}
F_{1 \_\mathrm{I}}= & -\frac{L_{1} B^{2}}{2 \mu_{0} \pi^{2} T_{1}}\left\{\left[\pi T_{1}\left(\pi T_{1}-8 h-2 a \pi\right)\right]\right. \\
& \left.+32 h^{2} \ln \left(1+\pi T_{1} / 4 h\right)\right\}, \\
F_{\mathrm{g} \_\mathrm{I}}= & \frac{h L_{1} B^{2}}{2 \mu_{0} \pi^{2} T_{1}^{2}}\left\{\left[\pi T_{1}\left(\pi T_{1}-8 h\right)\right]\right. \\
& \left.+32 h^{2} \ln \left(1+\pi T_{1} / 4 h\right)\right\} .
\end{aligned}\right.
$$

类似地, 可以得到区间 II 悬浮力 $F_{1 \_I I}$ 和导向力 $F_{\mathrm{g}_{\_} \mathrm{I}}$,

$$
\left\{\begin{aligned}
F_{1 \_ \text {II }}= & -\frac{L_{2} B^{2}}{2 \mu_{0} \pi^{2} T_{2}}\left\{\left[\pi T_{2}\left(\pi T_{2}-8 h-2 a \pi\right)\right]\right. \\
& \left.+32 h^{2} \ln \left(1+\pi T_{2} / 4 h\right)\right\}, \\
F_{\text {g_II }}= & \frac{h L_{2} B^{2}}{2 \mu_{0} \pi^{2} T_{2}^{2}}\left\{\left[\pi T_{2}\left(\pi T_{2}-8 h\right)\right]\right. \\
& \left.+32 h^{2} \ln \left(1+\pi T_{2} / 4 h\right)\right\} .
\end{aligned}\right.
$$

根据(4), (8)和(9)式, 得到一个模块提供的悬浮 力和导向力

$$
\left\{\begin{array}{l}
F_{1}=K_{\mathrm{m}} K_{\mathrm{L}} B^{2}, \\
F_{\mathrm{g}}=K_{\mathrm{m}} K_{\mathrm{G}} B^{2},
\end{array}\right.
$$

其中

$$
\left\{\begin{aligned}
K_{\mathrm{m}}= & \frac{1}{2 \mu_{0} \pi^{2}}, \\
K_{\mathrm{L}}= & -\frac{L_{1}}{T_{1}}\left(\left(\pi T_{1}\left(\pi T_{1}-8 h-2 a \pi\right)\right)+32 h^{2} \ln \left(1+\frac{\pi T_{1}}{4 h}\right)\right) \\
& -\frac{L_{2}}{T_{2}}\left(\left(\pi T_{2}\left(\pi T_{2}-8 h-2 a \pi\right)\right)+32 h^{2} \ln \left(1+\frac{\pi T_{2}}{4 h}\right)\right), \\
K_{\mathrm{G}}= & \frac{h L_{2}}{T_{2}^{2}}\left(\left(\pi T_{2}\left(\pi T_{2}-8 h\right)\right)+32 h^{2} \ln \left(1+\frac{\pi T_{2}}{4 h}\right)\right) \\
& -\frac{h L_{1}}{T_{1}^{2}}\left(\left(\pi T_{1}\left(\pi T_{1}-8 h\right)\right)+32 h^{2} \ln \left(1+\frac{\pi T_{1}}{4 h}\right)\right) .
\end{aligned}\right.
$$




\section{3 轨道超高条件下列车动力学平衡条件}

为了抵消磁悬浮列车通过水平弯道时的侧向离 心力, 轨道的外轨在弯道段具有一定的超高. 通常情 况下, 电磁铁的极面与轨道的极面总是保持平行的, 因此在弯道段列车相对水平面存在一个倾角, 角度 与轨道的曲线横坡角一致. 要保证列车安全通过, 列 车在超高的轨道上必须保持动力学平衡, 列车两侧 的模块提供的悬浮力必须满足列车的平衡条件.

不考虑空气弹簧长度的变化, 单转向架系统的 受力如图 5 所示, 其中 $M$ 为车体的质量, $F_{\mathrm{c}}$ 为车体受 到的离心力, $m$ 为模块自身的质量, $F_{1 \_\mathrm{a}}$ 和 $F_{1 \_\mathrm{b}}$ 分别为 两个模块提供的悬浮力, $F_{\mathrm{g}_{\mathrm{a}} \mathrm{a}}$ 和 $F_{\mathrm{g} \_\mathrm{b}}$ 分别为两个模块 提供的导向力, $f_{\mathrm{c} \_\mathrm{a}}$ 和 $f_{\mathrm{c} \_\mathrm{b}}$ 分别为两个模块受到的离心 力; $D$ 为车体重心到悬浮力作用线的距离, $d$ 为车体重 心到导向力作用线的距离, $\theta$ 为轨道的曲线横坡角. 各个力均以箭头所示方向为正方向, 力矩则以逆时 针为正方向.

设磁悬浮列车通过轨道水平弯道段时的离心加 速度为 $a_{\mathrm{c}}$, 则车体与两个模块受到的离心力分别为

$$
\left\{\begin{array}{l}
F_{\mathrm{c}}=M a_{\mathrm{c}}, \\
f_{\mathrm{c}_{-} \mathrm{a}}=f_{\mathrm{c}_{-} \mathrm{b}}=m a_{\mathrm{c}} .
\end{array}\right.
$$

根据力平衡条件, 可知

$$
\left\{\begin{aligned}
& F_{1_{-} \mathrm{a}}+F_{1_{-} \mathrm{b}}=(M+2 m) g \cos \theta \\
&+\left(f_{\mathrm{c}_{-} \mathrm{a}}+f_{\mathrm{c}_{-} \mathrm{b}}+F_{\mathrm{c}}\right) \sin \theta, \\
& F_{\mathrm{g}_{-} \mathrm{a}}+F_{\mathrm{g}_{-} \mathrm{b}}+\left(f_{\mathrm{c}_{-} \mathrm{a}}+f_{\mathrm{c}_{-} \mathrm{b}}+F_{\mathrm{c}}\right) \cos \theta \\
&=(M+2 m) g \sin \theta .
\end{aligned}\right.
$$

根据力矩平衡条件, 可知

$$
\begin{aligned}
& F_{1_{-} \mathrm{b}} D-F_{1_{-} \mathrm{a}} D+M g d \sin \theta+m g l_{\mathrm{a}}-m g l_{\mathrm{b}} \\
& -F_{\mathrm{c}} d \cos \theta+f_{\mathrm{c}_{-} \mathrm{a}} d_{\mathrm{a}}-f_{\mathrm{c}_{-} \mathrm{b}} d_{\mathrm{b}}=0 .
\end{aligned}
$$

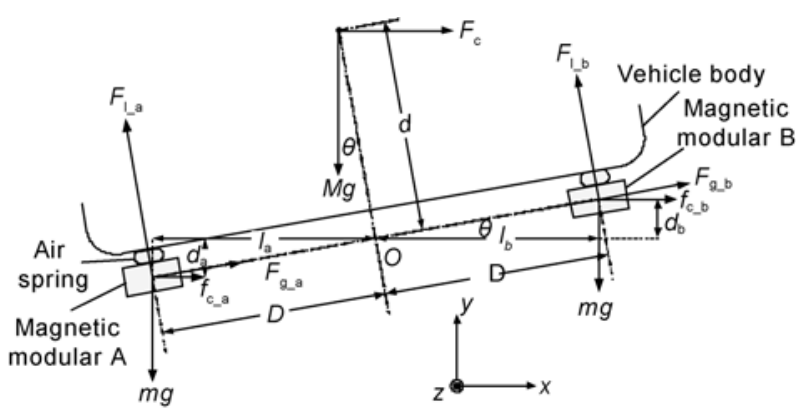

图 5 单转向架系统在水平弯道段的受力分析
根据图 5 中的几何关系, 有

$$
\left\{\begin{array}{l}
l_{\mathrm{a}}=l_{\mathrm{b}}=D \cos \theta, \\
d_{\mathrm{a}}=d_{\mathrm{b}}=D \sin \theta .
\end{array}\right.
$$

将(14)式代入到(13)式中, 结合(11)式, 整理可得

$$
\left(F_{1_{-} \mathrm{b}}-F_{1_{-} \mathrm{a}}\right) D+M\left(g \sin \theta-a_{\mathrm{c}} \cos \theta\right) d=0 .
$$

由(12)和(15)式, 得到单转向架一车体系统在弯 道段的动力学平衡方程

$$
\left\{\begin{aligned}
F_{1_{-} \mathrm{a}}= & \frac{1}{2}\left[(M+2 m)\left(g \cos \theta+a_{\mathrm{c}} \sin \theta\right)\right. \\
& \left.+\frac{d}{D} M\left(g \sin \theta-a_{\mathrm{c}} \cos \theta\right)\right], \\
F_{1_{-} \mathrm{b}}= & \frac{1}{2}\left[(M+2 m)\left(g \cos \theta+a_{\mathrm{c}} \sin \theta\right)\right. \\
& \left.-\frac{d}{D} M\left(g \sin \theta-a_{\mathrm{c}} \cos \theta\right)\right], \\
F_{\mathrm{g}_{-} \mathrm{a}}+ & F_{\mathrm{g}_{-} \mathrm{b}}=(M+2 m)\left(g \sin \theta-a_{\mathrm{c}} \cos \theta\right) .
\end{aligned}\right.
$$

列车以平衡时速 $V_{0}$ 运行时, 悬浮系统提供的导 向力的合力为零, 即

$$
F_{\mathrm{g}_{-} \mathrm{a}}+F_{\mathrm{g}_{-} \mathrm{b}}=0 .
$$

根据列车在轨道平行方向上的力平衡条件, 有

$$
\left(F_{\mathrm{c}}+f_{\mathrm{c}_{-} \mathrm{a}}+f_{\mathrm{c}_{-} \mathrm{b}}\right) \cos \theta=(M+2 m) g \sin \theta .
$$
有

设此时的离心加速度为 $a_{0}$, 则根据(11)和(18)式,

$$
a_{0}=g \tan \theta \text {. }
$$

将(17)和(19)式代入(16)式, 整理可得

$$
\left\{\begin{array}{l}
F_{1_{-} \mathrm{a}}=F_{1_{-} \mathrm{b}}=\frac{1}{2}(M+2 m)\left(g \cos \theta+a_{\mathrm{c}} \sin \theta\right), \\
F_{\mathrm{g}_{-} \mathrm{a}}+F_{\mathrm{g}_{-} \mathrm{b}}=0 .
\end{array}\right.
$$

可见, 当列车以平衡时速 $V_{0}$ 通过水平弯道时, 两个模块提供的悬浮力相等, 导向力合力为零.

磁悬浮列车轨道水平弯道通常为圆曲线, 设水 平弯道的半径为 $R$, 磁悬浮列车在弯道段的通过时速 为 $V$, 则离心加速度为

$$
a_{\mathrm{c}}=V^{2} / R \text {. }
$$

结合(20)式可知, 当列车的通过速度小于平衡时 速时, 离心加速度满足 $0 \leq a_{\mathrm{c}}<a_{0}$, , 两个模块提供的 导向力的合力指向弯道的外侧, 即 


$$
\left\{\begin{array}{l}
F_{1_{\_} \mathrm{a}}>F_{1_{-} \mathrm{b}}, \\
F_{\mathrm{g}_{-} \mathrm{a}}+F_{\mathrm{g}_{-} \mathrm{b}}>0 .
\end{array}\right.
$$

当列车的通过速度大于平衡时速时, 离心加速 度满足 $a_{\mathrm{c}}>a_{0}$, 悬浮系统提供的悬浮力和导向力应 满足

$$
\left\{\begin{array}{l}
F_{1_{-} \mathrm{b}}>F_{1_{-a}}, \\
F_{\mathrm{g}_{-} \mathrm{a}}+F_{\mathrm{g}_{-} \mathrm{b}}<0 .
\end{array}\right.
$$

设悬浮系统在磁场饱和的情况下提供的最大悬 浮力为 $F_{\mathrm{s}}$, 则在单转向架系统动力学平衡的条件下, 悬浮系统能够承载的最大车体质量 $M_{\max }$ 与最大悬浮 力 $F_{\mathrm{s}}$ 的关系为

$$
\begin{aligned}
M_{\text {max }}= & \left.\frac{2\left[F_{\mathrm{s}}-m\left(g \cos \theta+a_{\mathrm{c}} \sin \theta\right)\right]}{D\left(g \cos \theta+a_{\mathrm{c}} \sin \theta\right)} \quad \text { (当 } V=V_{0}\right), \\
M_{\text {max }}= & \frac{2 D\left[F_{\mathrm{s}}-m\left(g \cos \theta+a_{\mathrm{c}} \sin \theta\right)\right]}{D\left(g \cos \theta+a_{\mathrm{c}} \sin \theta\right)+d\left(g \sin \theta-a_{\mathrm{c}} \cos \theta\right)} \\
& \left(\text { 当 } V<V_{0}\right), \\
M_{\text {max }}= & \frac{2 D\left[F_{\mathrm{s}}-m\left(g \cos \theta+a_{\mathrm{c}} \sin \theta\right)\right]}{D\left(g \cos \theta+a_{\mathrm{c}} \sin \theta\right)-d\left(g \sin \theta-a_{\mathrm{c}} \cos \theta\right)} \\
& \left(\text { 当 } V>V_{0}\right) .
\end{aligned}
$$

综上所述, 当 $V=V_{0}$ 时, $M_{\max }$ 最大, 此时悬浮系统 提供的导向力的合力为零; 其他两种情况下, 系统可 承载的最大质量相对较小.

\section{4 悬浮系统弯道承载能力的计算与分析}

通常情况下, 列车的运行速度不大于平衡时速, 即 $V \leq V_{0}$. 此时, 悬浮系统提供的导向力指向弯道外 侧. 根据前面的分析可知,

1) 运行速度 $V$ 越小, 需要悬浮系统提供的导向 力越大, 而悬浮系统可以提供悬浮力越小; 当列车静 止在弯道上时, 需要悬浮系统提供的导向力最大, 悬 浮系统可以提供的悬浮力最小.

2) 悬浮间隙 $h$ 越大, 悬浮系统可以提供的悬浮 力越小; 当列车从降落状态开始起浮时, 悬浮系统可 以提供的悬浮力最小.

3) 要保持列车静止悬浮在弯道上时的动力学平 衡, 靠弯道内侧的模块提供的悬浮力较大, 并将首先 达到磁场饱和.
综合上述三点可知, 当列车静止在弯道上并从 降落状态开始起浮时, 悬浮系统能够提供的电磁力 最小, 此时悬浮系统在磁场饱和的情况下能够承载 的车体质量就是列车的最大承载能力. 根据(19)和 (25)式以及条件 $a_{\mathrm{c}}=0$, 有

$$
M_{\text {max }}=\frac{2 D\left(K_{\mathrm{m}} K_{\mathrm{L}} B_{\mathrm{a}}^{2}-m g \cos \theta\right)}{D g \cos \theta+d g \sin \theta} .
$$

(27)式反映了列车在弯道的最大承载能力与弯道半 径 $R$ 、轨道超高下的曲线横坡角 $\theta$ 、轨道跨距 $2 D$ 及车 体重心高度 $d$ 之间的关系, 下面通过算例分别加以讨 论. 设降落状态时悬浮间隙为 $0.02 \mathrm{~m}$, 悬浮电磁铁采 用 $\mathrm{A} 3$ 钢作为铁芯材料, 饱和磁感应强度为 $1.25 \mathrm{~T}$, 每个模块的质量为 $860 \mathrm{~kg}$, 整列磁悬浮列车包含 4 个 转向架.

1) 最大承载质量与弯道半径、轨道曲线横坡角 的关系. 设轨道跨距为 $2 \mathrm{~m}$, 车体重心高度为 $0.6 \mathrm{~m}$, 根据(27)式计算列车的最大承载能力, 结果如表 1 所示.

2) 最大承载质量与轨道跨距、车体重心的关系. 设弯道半径为 $100 \mathrm{~m}$, 轨道曲线横坡角为 $10^{\circ}$. 根据 (27)式计算列车的最大承载能力, 结果如表 2 所示.

通过上述方法可计算磁悬浮列车在平直轨道上 的最大承载能力

$$
M_{\text {max }}=4\left(\frac{S B^{2}}{\mu_{0} g}-2 m\right)=40.87 .
$$

表 1 不同弯道半径和轨道横坡角条件下列车的最大承载 能力(单位: $\mathbf{t}$ )

\begin{tabular}{ccccc}
\hline$R(\mathrm{~m})$ & $\theta=5^{\circ}$ & $\theta=10^{\circ}$ & $\theta=15^{\circ}$ & $\theta=20^{\circ}$ \\
\hline 75 & 34.22 & 33.02 & 32.19 & 31.68 \\
100 & 36.07 & 34.80 & 33.92 & 33.37 \\
125 & 37.02 & 35.72 & 34.80 & 34.24 \\
150 & 37.57 & 36.25 & 35.32 & 34.75 \\
\hline
\end{tabular}

表 2 不同轨道跨距和车体重心高度条件下列车的最大承 载能力(单位: $\mathbf{t}$ )

\begin{tabular}{ccccc}
\hline$D(\mathrm{~m})$ & $d=0.6 \mathrm{~m}$ & $d=0.8 \mathrm{~m}$ & $d=1.0 \mathrm{~m}$ & $d=1.2 \mathrm{~m}$ \\
\hline 0.8 & 33.99 & 32.72 & 31.53 & 30.43 \\
0.9 & 34.44 & 33.27 & 32.18 & 31.16 \\
1.0 & 34.80 & 33.73 & 32.72 & 31.76 \\
1.1 & 35.11 & 34.11 & 33.17 & 32.28 \\
\hline
\end{tabular}


根据表 1 和表 2 可得到如下结论.

1) 磁悬浮列车在弯道段的最大承载能力小于在 平直轨道上的承载能力.

2) 磁悬浮列车的最大弯道承载能力随弯道半径 增大而增大, 随轨道曲线横坡角增大而减小. 如果因 为工程方面的原因希望转弯半径小而不影响弯道的 承载能力, 可以适当减小轨道的曲线横坡角. 而根据 (19)和(21)式, 有

$$
V_{0}=\sqrt{R g \tan \theta},
$$

转弯半径和轨道曲线横坡角都减小的直接后果就是 弯道的平衡时速降低.

3) 轨道跨距越大, 列车的弯道承载能力越大, 因此在设计车辆时可以适当考虑增加车辆的宽度以 提高列车的承载能力.

4) 车体重心位置越高, 列车的弯道承载能力越 小. 磁悬浮列车满载时, 车体的重心相对空载情况会 有所提高, 在规定列车的最大承载质量时必须考虑 该因素.
5) 列车承载能力的计算公式(27)和(28), 是从电 磁铁所能提供最大电磁力的角度得到的理论值, 仅 仅考虑了电磁铁磁场饱和特性的影响, 没有考虑其 他工程因素的制约, 例如: 车体刚度、轨道刚度和转 向架刚度等. 因此, 列车的实际承载能力要小于 (27) 和(28)式的计算结果.

6) 表 1 和 2 的计算结果，是在横向止档没有提 供辅助导向力的情况下得到的. 若计算结果表明悬 浮模块的偏移超出了横向止档的位置, 即横向止档 提供辅助导向力, 此时列车承载能力不能根据(27)和 (28)式计算得到.

\section{5 结论}

常导低速磁悬浮列车在弯道段的承载能力是提 高其承载能力的瓶颈. 本文利用磁通管法, 分析影响 磁悬浮列车弯道承载能力的几个主要因素, 得出列 车承载能力的定量计算公式. 为磁悬浮列车安全可 靠运行和今后的整体结构设计提供了理论依据.

\section{参考文献}

1 刘德生. EMS 型低速磁浮列车模块悬浮系统控制技术研究. 博士学位论文. 长沙: 国防科技大学, 2006

2 Liu D S, Li J, Chang W S. Design of nonlinear decoupling controller for double-electromagnet suspension system. Acta Auto Sin, 2006, 32(3): $321-328$

3 郑永成, 王洋, 何建国, 等. 基于磁场分割的磁导计算与磁路设计. 机械与电子, 2006, 7: 11-16

4 金志颖, 杨仕友, 倪光正. EMS 型磁浮列车电磁系统动态电磁场的有限元分析及其悬浮与车引力特性的研究. 中国电机工程学报, 2004, 24(10): 133-137

5 徐强, 廖启征, 魏世民, 等. 平动式啮合电机的非线性等效磁路模型. 电机与控制学报, 2008, 2: 126一 131

6 张曙光. 高速列车转向架载荷谱测试与建立方法的研究. 中国科学 $\mathrm{E}$ 辑: 技术科学, 2008, 38(11): 1805-1814 